$1-1-2007$

\title{
Periodic Solution to The Time-Inhomogeneous Multi-Server Poisson Queue
}

Barbara Haas Margolius

Cleveland State University, b.margolius@csuohio.edu

Follow this and additional works at: https://engagedscholarship.csuohio.edu/scimath_facpub

Part of the Mathematics Commons

How does access to this work benefit you? Let us know!

\section{Repository Citation}

Margolius, Barbara Haas, "Periodic Solution to The Time-Inhomogeneous Multi-Server Poisson Queue" (2007). Mathematics Faculty Publications. 215.

https://engagedscholarship.csuohio.edu/scimath_facpub/215

This Article is brought to you for free and open access by the Mathematics and Statistics Department at EngagedScholarship@CSU. It has been accepted for inclusion in Mathematics Faculty Publications by an authorized administrator of EngagedScholarship@CSU. For more information, please contact library.es@csuohio.edu. 


\title{
Periodic solution to the time-inhomogeneous multi-server Poisson queue
}

\author{
B.H. Margolius
}

\section{Introduction}

In many queueing applications, arrival rates and number of servers vary periodically. Examples include call centers, emergency dispatching, and retail establishments. For such queues with the average arrival rate less than the average effective service rate, periodic asymptotic probabilities $p_{j}(t)$, the probability of $j$ in the queue at time $t, t \in[0, T)$ where $T$ is the length of the period, exist. We present a method for computing these probabilities using one period of data. We also derive formulae for the rising factorial moments of the number in the queue as a function of time within the period. This is an extension of results obtained for the transient solution for the time-dependent Poisson queue given in [10]. As noted in [15] and references cited there, computational methods and approximation techniques involved in time-inhomogeneous queueing problems have long been regarded as challenging. In this paper, we present a relatively simple method for computing these quantities for queues with periodically varying rates via the numerical solution of integral equations covering only one time period.

Literature surveys in the area of queues with time-varying rates are available in [12,9]. Treatment of the singleserver Poisson queue with time-varying arrival rate and constant service dates back at least to A.N. Kolmogorov's 
paper on the waiting time distribution in 1931 [7,8]. Additional references and an overview of many mathematical tools related to queues with time-varying rates is available in [11].

Early work in the area of time-dependent Poisson queues with periodic rates includes work by Hasofer for the single-server queue with general service [3,4]. In Do Le Minh's paper [12] treating the discrete-time queue with time-varying compound Poisson input and general service, he describes Hasofer's work as elaborate, but having limited usefulness in computational applications. More recent work in this area includes Breuer's work on the $B M A P / P H / c$ periodic queue.

We assume a pre-emptive discipline so that when the number of servers decreases, customers who were in service return to the queue. This contrasts with the exhaustive discipline in which the server completes service of customers being served before leaving. See [5] for recent work on an approach for modeling the $M_{t} / M / c_{t}$ queue with the exhaustive discipline.

\section{The family of generating functions}

Consider a queueing system with time-varying periodic arrival and service rates, $\lambda(t)$, and $\mu(t)$, respectively, and time-varying periodic number of servers, $c(t)$. For ease of exposition, we consider the case where the period is of length 1. $\hat{c}=\max _{t} c(t)$ is the maximum number of servers in the period.

Define $P_{i, j}(s, t)$ as the probability that the system is in state $j$ at time $t$ given that it was in state $i$ at time $s$ and

$$
p_{j}(t)=\lim _{n \rightarrow \infty} P_{i, j}(s, n+t), \quad 0 \leqslant t<1, \quad n \text { integer. }
$$

For each $t$ in $[0,1), p_{j}(t), j=0,1,2, \ldots$ is the periodic asymptotic distribution for that time within the period. This family of distributions will exist if $\int_{0}^{1} \lambda(t) \mathrm{d} t<\int_{0}^{1} c(t) \mu(t) \mathrm{d} t$. For a proof of this result, see [1].

We can write the Chapman-Kolmogorov forward differential equations for a time-dependent multi-server queue with time-varying number of servers as (since $\left.p_{-1}(t)=0\right)$

$$
\dot{p}_{n}(t)=-p_{n}(t)(\lambda(t)+(n \wedge c(t)) \mu(t))+p_{n-1}(t) \lambda(t)+p_{n+1}(t)((n+1) \wedge c(t)) \mu(t),
$$

where $x \wedge y=\min \{x, y\}$. We will also use the notation, $x \vee y=\max \{x, y\}$.

Recall that the limiting probabilities for the $M / M / c$ queue satisfy the equations $\lambda p_{n}=(n \wedge c) \mu p_{n+1}$. For the periodic $M_{t} / M_{t} / c_{t}$ queue we have an analogous result:

$$
\int_{t-1}^{t} \lambda(\eta) p_{n-1}(\eta) \mathrm{d} \eta=\int_{t-1}^{t}(n \wedge c(\eta)) \mu(\eta) p_{n}(\eta) \mathrm{d} \eta
$$

for all $n \geqslant 1$ and all $t$. To prove this result, simply integrate (1) and use periodicity and mathematical induction. This result is a corollary to the following more general statement for birth-death processes with periodic rates:

Theorem 1. For a birth-death process with periodic rates for which a periodic asymptotic distribution exists,

$$
\int_{t-1}^{t} \lambda_{n-1}(\eta) p_{n-1}(\eta) \mathrm{d} \eta=\int_{t-1}^{t} \mu_{n}(\eta) p_{n}(\eta) \mathrm{d} \eta
$$

for all $n \geqslant 1$ and all $t$. 
We define a family of generating functions $P_{z}^{(k)}(s, t)=q_{k}(t)+\sum_{n=1}^{\infty} p_{n+k}(t) z^{n}$ where $q_{k}(t)=\sum_{j=0}^{k} p_{j}(t)$. Using (1) we obtain the following differential equation for $P_{z}^{(k)}(s, t)$ :

$$
\begin{aligned}
\frac{\partial}{\partial t} P_{z}^{(k)}(s, t)= & \left((z-1) \lambda(t)+\left(z^{-1}-1\right) c(t) \mu(t)\right)\left(P_{z}^{(k)}(s, t)-q_{k-1}(t)\right)+\mu(t)\left(1-z^{-1}\right) \\
& \times \sum_{j=k}^{c(t)}(c(t)-j) p_{j}(t) z^{j-k}+\mu(t)(k \wedge c(t))\left(1-z^{-1}\right) p_{k}(t),
\end{aligned}
$$

where for $k>\hat{c}$, we define $\sum_{j=k}^{\hat{c}} f(j)$ as being equal to zero. This differential equation has the solution,

$$
\begin{aligned}
P_{z}^{(k)}(s, t)= & \int_{s}^{t} \Phi_{z}(\eta, t)\left[\mu(\eta)\left(1-z^{-1}\right) \sum_{j=k}^{c(\eta)}(c(\eta)-j) p_{j}(\eta) z^{j-k}+\lambda(\eta) p_{k-1}(\eta)-(c(\eta) \wedge k) \mu(\eta) z^{-1} p_{k}(\eta)\right] \\
& \times \mathrm{d} \eta+q_{k-1}(t)-q_{k-1}(s) \Phi_{z}(s, t)+\Phi_{z}(s, t) P_{z}^{(k)}(s, s),
\end{aligned}
$$

where

$$
\Phi_{z}(s, t)=\exp \left\{\int_{s}^{t}\left(z \lambda(\eta)-(\lambda(\eta)+c(\eta) \mu(\eta))+c(\eta) \mu(\eta) z^{-1}\right) \mathrm{d} \eta\right\} .
$$

Define

$$
\tilde{I}_{n}(s, t)= \begin{cases}\sum_{k=0}^{\infty} \frac{\Lambda^{n+k}(s, t)}{(n+k) !} \frac{M^{k}(s, t)}{k !} \mathrm{e}^{-\Lambda(s, t)-M(s, t)} & \text { if } n \geqslant 0, \\ \sum_{k=0}^{\infty} \frac{\Lambda^{k}(s, t)}{k !} \frac{M^{k-n}(s, t)}{(k-n) !} \mathrm{e}^{-\Lambda(s, t)-M(s, t)} & \text { if } n<0,\end{cases}
$$

where $\Lambda(s, t)=\int_{s}^{t} \lambda(\eta) \mathrm{d} \eta$ and $M(s, t)=\int_{s}^{t} c(\eta) \mu(\eta) \mathrm{d} \eta$. Note that $\tilde{I}_{n}(s, t)$ is the probability of $n$ more steps to the right than to the left during the time interval from $s$ to $t$ for a random walk with time-dependent rates $\lambda(t)$ for steps to the right and $c(t) \mu(t)$ for steps to the left. The $\tilde{I}_{n}(s, t)$ are related to the modified Bessel functions $I_{n}(x)$. Recall that the $n$th modified Bessel function $I_{n}(x)=(x / 2)^{n} \sum_{k=0}^{\infty}\left((x / 2)^{2 k} /(n+k) ! k !\right)$, so

$$
\tilde{I}_{n}(s, t)=\left(\frac{\Lambda(s, t)}{M(s, t)}\right)^{n / 2} I_{n}(2 \sqrt{\Lambda(s, t) M(s, t)}) \mathrm{e}^{-\Lambda(s, t)-M(s, t)}
$$

and $\tilde{I}_{0}(t, t)=I_{0}(0)=1$. One can show that $\Phi_{z}(s, t)=\sum_{n=-\infty}^{\infty} z^{n} \tilde{I}_{n}(s, t)$, see, for example, [16] Appendix B.

Consider $s=t-1$. Recall that $\mu(t), \lambda(t)$ and $c(t)$ are each periodic with period 1 . Then we have

$$
\begin{aligned}
P_{z}^{(k)}(t-1, t)= & {\left[1-\Phi_{z}(t-1, t)\right]^{-1} \int_{t-1}^{t} \Phi_{z}(\eta, t)\left[\mu(\eta)\left(1-z^{-1}\right) \sum_{j=k}^{c(\eta)}(c(\eta)-j) p_{j}(\eta) z^{j-k}+\lambda(\eta) p_{k-1}(\eta)\right.} \\
& \left.-(k \wedge c(\eta)) \mu(\eta) z^{-1} p_{k}(\eta)\right] \mathrm{d} \eta+q_{k-1}(t) .
\end{aligned}
$$


In particular, when $k=\hat{c}-1$, then (2) simplifies to

$$
\begin{aligned}
P_{z}^{(\hat{c}-1)}(t-1, t)= & {\left[1-\Phi_{z}(t-1, t)\right]^{-1} \int_{t-1}^{t} \Phi_{z}(\eta, t)\left[\lambda(\eta) p_{\hat{c}-2}(\eta)+((0 \vee c(\eta)-\hat{c}+1)\right.} \\
& \left.\left.-c(\eta) z^{-1}\right) \mu(\eta) p_{\hat{c}-1}(\eta)\right] \mathrm{d} \eta+q_{\hat{c}-2}(t),
\end{aligned}
$$

for $k>\hat{c}-1$,

$$
P_{z}^{(k)}(t-1, t)=\frac{\int_{t-1}^{t} \Phi_{z}(\eta, t)\left[\lambda(\eta) p_{k-1}(\eta)-(k \wedge c(\eta)) \mu(\eta) z^{-1} p_{k}(\eta)\right] \mathrm{d} \eta}{1-\Phi_{z}(t-1, t)},
$$

and for $k=0$, we have

$$
P_{z}^{(0)}(t-1, t)=\frac{\int_{t-1}^{t} \Phi_{z}(\eta, t)\left[\mu(\eta)\left(1-z^{-1}\right) \sum_{j=k}^{c(\eta)}(c(\eta)-j) p_{j}(\eta) z^{j-k}\right] \mathrm{d} \eta}{1-\Phi_{z}(t-1, t)} .
$$

Consider $\left[1-\Phi_{z}(t-1, t)\right]^{-1}$. We proceed with a formal study of this series. Note that if $\int_{t-1}^{t} \lambda(s) \mathrm{d} s<\int_{t-1}^{t} c(s) \mu(s) \mathrm{d} s$, then for $|z|<1, \Phi_{z}(t-1, t)>1$. Nonetheless, formally we may write $\left[1-\Phi_{z}(t-1, t)\right]^{-1}=\sum_{k=0}^{\infty} \Phi_{z}^{j}(t-1, t)$. See $[14$, p. 30], for a discussion of the formal study of generating functions. Now

$$
\Phi_{z}^{j}(t-1, t)=\exp \left\{\int_{t-1}^{t}\left(z j \lambda(\eta)-(j \lambda(\eta)+j c(\eta) \mu(\eta))+j c(\eta) \mu(\eta) z^{-1}\right) \mathrm{d} \eta\right\},
$$

and so $\Phi_{z}^{j}(t, t-1)=\sum_{n=-\infty}^{\infty} z^{n} \tilde{I}_{n}^{(j)}(t-1, t)$, where

$$
\tilde{I}_{n}^{(j)}(t-1, t)= \begin{cases}\sum_{k=0}^{\infty} \frac{(j \Lambda(t-1, t))^{n+k}}{(n+k) !} \frac{(j M(t-1, t))^{k}}{k !} \mathrm{e}^{-j \Lambda(t-1, t)-j M(t-1, t)} & \text { if } n \geqslant 0, \\ \sum_{k=0}^{\infty} \frac{(j \Lambda(t-1, t))^{k}}{k !} \frac{(j M(t-1, t))^{k-n}}{(k-n) !} \mathrm{e}^{-j \Lambda(t-1, t)-j M(t-1, t)} & \text { if } n<0 .\end{cases}
$$

This is the probability a time-dependent random walk takes $n$ more steps to the right than to the left during the time interval $[t-1, t)$ if a step to the right occurs at rate $j \lambda(t)$ and a step to the left occurs at rate $j c(t) \mu(t)$. It can also be regarded as the probability that a time-dependent random walk takes $n$ more steps to the right than to the left during the time interval $[t-j, t)$ if a step to the right occurs at rate $\lambda(t)$ and a step to the left occurs at rate $c(t) \mu(t)$, for $j=0,1,2, \ldots$.

Note also that $\Phi_{z}^{j}(t-1, t) \Phi_{z}(\eta, t)=\sum_{n=-\infty}^{\infty} z^{n} \tilde{I}_{n, j}(\eta, t)$, where

$$
\tilde{I}_{n, j}(\eta, t)= \begin{cases}\sum_{k=0}^{\infty} \frac{(\Lambda(\eta, t)+j \Lambda(t-1, t))^{n+k}}{(n+k) !} \frac{(M(\eta, t)+j M(t-1, t))^{k}}{k !} \mathrm{e}^{-j \Lambda(t-1, t)-j M(t-1, t)-\Lambda(\eta, t)-M(\eta, t)} & \text { if } n \geqslant 0, \\ \sum_{k=0}^{\infty} \frac{(\Lambda(\eta, t)+j \Lambda(t-1, t))^{k}}{k !} \frac{(M(\eta, t)+j M(t-1, t))^{k-n}}{(k-n) !} \mathrm{e}^{-j \Lambda(t-1, t)-j M(t-1, t)-\Lambda(\eta, t)-M(\eta, t)} & \text { if } n<0 .\end{cases}
$$

The coefficients on $z^{n-k}$ in (3) and (4) yield the following lemma:

Lemma 1. For $n \geqslant k \geqslant \hat{c}$,

$$
p_{n}(t)=\int_{t-1}^{t}\left(H_{n-k}(\eta, t) \lambda(\eta) p_{k-1}(\eta)-H_{n-k+1}(\eta, t) c(\eta) \mu(\eta) p_{k}(\eta)\right) \mathrm{d} \eta
$$


and for $n \geqslant \hat{c}-1$,

$$
\begin{aligned}
p_{n}(t)= & \int_{t-1}^{t}\left(H_{n-\hat{c}+1}(\eta, t)\left(\lambda(\eta) p_{\hat{c}-2}(\eta)+(0 \vee c(\eta)-\hat{c}+1) \mu(\eta) p_{\hat{c}-1}(\eta)\right)\right. \\
& \left.-H_{n-\hat{c}+2}(\eta, t) c(\eta) \mu(\eta) p_{\hat{c}-1}(\eta)\right) \mathrm{d} \eta,
\end{aligned}
$$

where $H_{n}(s, t)=\sum_{j=0}^{\infty} \tilde{I}_{n, j}(s, t)$.

In the Appendix, we show that $H_{n}(\eta, t)$ may be approximated with arbitrary precision by a finite sum of these random walk probabilities, $\tilde{I}_{n, j}(\eta, t)$.

Note the particular case of Eq. (6) for which $n=\hat{c}-1$. This gives an integral equation for $p_{\hat{c}-1}(t)$ in terms of itself and $p_{\hat{c}-2}(t)$. Using the system of differential equations given in (1), we may find an expression for $p_{\hat{c}-2}(t)$ in terms of $p_{\hat{c}-1}(t)$. More generally, we may express $p_{j}(t)$ in terms of an integral equation in $p_{\hat{c}-1}(t)$ for $j<\hat{c}-1$. We can then use the resulting equation, to solve for $p_{\hat{c}-1}(t)$ numerically.

From (1), we have $\dot{\mathbf{p}}(t)=\mathbf{p}(t) \mathbf{A}(t)+(c(t) \wedge \hat{c}-1) \mu(t) p_{\hat{c}-1}(t) \mathbf{e}_{\hat{c}-1}$ where $\mathbf{e}_{\hat{c}-1}$ is a row vector of length $\hat{c}-1$ with zeros in the first $\hat{c}-2$ places and a one in the last place, $\mathbf{p}(t)$ is the row vector $\mathbf{p}(t)=\left(p_{0}(t), p_{1}(t), \ldots, p_{\hat{c}-2}(t)\right)$ and

$$
\begin{aligned}
& A_{i, i}(t)=-\lambda(t)-(i \wedge c(t)) \mu(t), \\
& A_{i, i+1}(t)=\lambda(t), \\
& A_{i, i-1}(t)=(i \wedge c(t)) \mu(t),
\end{aligned}
$$

$A_{i, j}(t)=0$, otherwise, and rows and columns are indexed beginning at zero.

Solving for $\mathbf{p}(t)$, we have $\mathbf{p}(t)=\int_{s}^{t}(c(v) \wedge \hat{c}-1) \mu(v) p_{\hat{c}-1}(v) \mathbf{e}_{\hat{c}-1} \mathbf{U}(v, t) \mathrm{d} v+\mathbf{p}(s) \mathbf{U}(s, t)$, where $\mathbf{U}(s, t)$ is an evolution operator such that $\mathbf{U}(t, t)=\mathbf{I}$ (the identity operator) and $(\partial / \partial t) \mathbf{U}(s, t)=\mathbf{U}(s, t) \mathbf{A}(t)$. $\mathbf{U}(s, t)$ can be solved for numerically using techniques for solving linear systems of ordinary differential equations. For each fixed $s$ and $t, \mathbf{U}(s, t)$ is a square matrix whose components we index from zero to $\hat{c}-2$. For $s=t-1$,

$$
\mathbf{p}(t)=\int_{t-1}^{t}(c(v) \wedge \hat{c}-1) \mu(v) p_{\hat{c}-1}(v) \mathbf{e}_{\hat{c}-1} \mathbf{U}(v, t) \mathrm{d} v(\mathbf{I}-\mathbf{U}(t-1, t))^{-1} .
$$

Let $\mathbf{G}(s, t)=\mathbf{U}(s, t)(\mathbf{I}-\mathbf{U}(t-1, t))^{-1}$, then for $j=0,1, \ldots, \hat{c}-2$,

$$
p_{j}(t)=\int_{t-1}^{t}(c(v) \wedge \hat{c}-1) \mu(v) p_{\hat{c}-1}(v) \mathbf{G}_{\hat{c}-2, j}(v, t) \mathrm{d} v=\int_{t-1}^{t} \mu(v) p_{\hat{c}-1}(v) F_{j}(v, t) \mathrm{d} v,
$$

where $F_{j}(v, t)=(c(v) \wedge \hat{c}-1) \mathbf{G}_{\hat{c}-2, j}(v, t)$.

Setting $n=\hat{c}-1$ in (6), substituting for $p_{\hat{c}-2}(t)$, using periodicity and changing the order of integration yields the following integral equation for $p_{\hat{c}-1}(t)$ :

$$
\begin{aligned}
p_{\hat{c}-1}(t)= & \int_{t-1}^{t} \mu(\eta) p_{\hat{c}-1}(\eta)\left[\left((0 \vee c(\eta)-\hat{c}+1) H_{0}(\eta, t)-c(\eta) H_{1}(\eta, t)\right)\right. \\
& \left.+\int_{t-1}^{\eta} F_{\hat{c}-2}(\eta-1, v) \lambda(v) H_{0}(v, t) \mathrm{d} v+\int_{\eta}^{t} F_{\hat{c}-2}(\eta, v) \lambda(v) H_{0}(v, t) \mathrm{d} v\right] \mathrm{d} \eta .
\end{aligned}
$$

The lemma and Eqs. (8) and (9) yield the following theorem for the $p_{n}(t)$.

Theorem 2. The periodic asymptotic distribution for the multi-server Poisson queue with periodic rates may be written in terms of the probability of $\hat{c}-1$ in the system where $\hat{c}$ is the maximum number of servers during the 
period. This probability is given in terms of the following Volterra equation of the second kind:

$$
\begin{aligned}
p_{\hat{c}-1}(t)= & \int_{t-1}^{t} \mu(\eta) p_{\hat{c}-1}(\eta)\left[\left((0 \vee c(\eta)-\hat{c}+1) H_{0}(\eta, t)-c(\eta) H_{1}(\eta, t)\right)\right. \\
& \left.+\int_{t-1}^{\eta} F_{\hat{c}-2}(\eta-1, v) \lambda(v) H_{0}(v, t) \mathrm{d} v+\int_{\eta}^{t} F_{\hat{c}-2}(\eta, v) \lambda(v) H_{0}(v, t) \mathrm{d} v\right] \mathrm{d} \eta .
\end{aligned}
$$

For $0 \leqslant n \leqslant \hat{c}-2$ we have

$$
p_{n}(t)=\int_{t-1}^{t}(c(v) \wedge \hat{c}-1) \mu(v) p_{\hat{c}-1}(v) \mathbf{G}_{\hat{c}-2, n}(v, t) \mathrm{d} v=\int_{t-1}^{t} \mu(v) p_{\hat{c}-1}(v) F_{n}(v, t) \mathrm{d} v,
$$

and for $n \geqslant \hat{c}-1$,

$p_{n}(t)=\int_{t-1}^{t}\left(H_{n-\hat{c}+1}(\eta, t)\left(\lambda(\eta) p_{\hat{c}-2}(\eta)+(0 \vee c(\eta)-\hat{c}+1) \mu(\eta) p_{\hat{c}-1}(\eta)\right)-H_{n-\hat{c}+2}(\eta, t) c(\eta) \mu(\eta) p_{\hat{c}-1}(\eta)\right) \mathrm{d} \eta$.

We may solve Eq. (9) numerically to get $C p_{\hat{c}-1}(t)$ for some constant $C$.

Since $\lim _{z \rightarrow 1} P_{z}^{(k)}(s, t)=1$, we have for $k=0$, by l'Hôpital's rule,

$$
M(t-1, t)-\Lambda(t-1, t)=\int_{t-1}^{t} \mu(\eta) \sum_{j=0}^{\hat{c}}(0 \vee c(\eta)-j) p_{j}(\eta) \mathrm{d} \eta .
$$

Given $C p_{\hat{c}-1}(t)$, to find $C$, we use (10) and follow the same sequence of steps as in the derivation of (9) to obtain

$$
\begin{aligned}
C= & (M(t-1, t)-\Lambda(t-1, t))^{-1} \int_{t-1}^{t} \mu(\eta) C p_{\hat{c}-1}(\eta)\left[(0 \vee c(\eta)-\hat{c}+1)+\int_{t-1}^{\eta} \sum_{j=0}^{\hat{c}-2} F_{j}(\eta-1, v)\right. \\
& \left.\times \mu(v)(0 \vee c(v)-j) \mathrm{d} v+\int_{\eta}^{t} \sum_{j=0}^{\hat{c}-2} F_{j}(\eta, v) \mu(v)(0 \vee c(v)-j) \mathrm{d} v\right] \mathrm{d} \eta .
\end{aligned}
$$

Solving these integral equations involves discretizing the functions, applying numerical integration weights, and solving a system of linear equations. We solve for a vector of values for $p_{\hat{c}-1}(t)$ that satisfy the discretized versions of both Eqs. (9) and (11). Given this numerical representation of $p_{\hat{c}-1}(t)$ we can apply (7) to compute $p_{j}(t)$ for $j<\hat{c}-1$ and we may substitute $p_{\hat{c}-1}(t)$ and $p_{\hat{c}-2}(t)$ into (6) to obtain the rest ofthe distribution.

When the number of servers is large, the system of differential equations that must be solved to compute the evolution operator $\mathbf{U}(s, t)$ will also become large and this technique will become impractical except for systems with special characteristics. As the maximum number of servers during the period, $\hat{c}$, tends to infinity, because the method relies on being able to compute the evolution operator for the first $\hat{c}-1$ states, the method becomes inapplicable.

\section{Moments}

In this section, we provide formulae for the queue length process $\{Q(t), t \geqslant 0\}$ with initial distribution given by $P\{Q(0)=n\}=p_{n}(0)$, where $p_{n}(0)$ are the periodic asymptotic probabilities for the beginning of the period. We 
define the functions: $q_{n}^{(m)}(t)=\sum_{j=n}^{\infty} q_{n}^{(m-1)}(t)$ for $m=0,1,2, \ldots$ and $n \in \mathbb{Z}$ with $q_{n}^{(0)}(t)=p_{n}(t)$. Then $q^{(1)}(t)=1$, $q_{0}^{(2)}(t)=E[Q(t)]+1$ and more generally, for $m \geqslant 1$,

$$
\begin{aligned}
q_{0}^{(m+1)}(t) & =E[(Q(t)+1)(Q(t)+2) \cdots(Q(t)+m)] / m ! \\
& =\frac{1}{m !} \sum_{k=0}^{m}(-1)^{m-k} s(m+1, k) E\left[Q^{k}(t)\right],
\end{aligned}
$$

where the $s(k, j)$ are the Stirling numbers of the first kind [13]. The rising factorial [6] is given by the formula:

$$
x^{\bar{m}}=x(x+1) \cdots(x+m-1) .
$$

The function $q_{0}^{(m+1)}(t)=E\left[(Q(t)+1)^{\bar{m}}\right]$, gives the expectation of the rising factorial of $Q(t)+1$, which we shall refer to as the $m$ th rising factorial moment.

In what follows, we also require the quantities $q_{-1}^{(m)}(t)$. Since $q_{-1}^{(0)}(t)=p_{-1}(t)=0, q_{-1}^{(1)}(t)=q_{0}^{(1)}(t)=1$. From the definition of $q_{n}^{(m)}(t), q_{-1}^{(m)}(t)=q_{0}^{(m)}(t)+q_{-1}^{(m-1)}(t)$, so for $m>1$,

$$
\begin{aligned}
q_{-1}^{(m)}(t) & =1+\sum_{j=1}^{m-1} E\left[(Q(t)+1)^{\bar{j}}\right] \\
& =1+\sum_{j=1}^{m-1} E\left[\prod_{k=1}^{j}(Q(t)+k)\right] / j ! \\
& =\frac{E[(Q(t)+2)(Q(t)+3) \cdots(Q(t)+m)]}{(m-1) !} .
\end{aligned}
$$

The functions $q_{n}^{(m)}(t)$ satisfy systems of differential equations similar to those for $q_{n}^{(0)}(t)=p_{n}(t)$ given in (1). For $n \geqslant 0$ we have:

$$
\dot{q}_{n}^{(1)}(t)=\lambda(t) q_{n-1}^{(1)}(t)-(\lambda(t)+c(t) \mu(t)) q_{n}^{(1)}(t)+c(t) \mu(t) q_{n+1}^{(1)}(t)+\mu(t)((c(t)-n) \vee 0) p_{n}(t),
$$

and for $m>1$,

$\dot{q}_{n}^{(m)}(t)=\lambda(t) q_{n-1}^{(m)}(t)-(\lambda(t)+c(t) \mu(t)) q_{n}^{(m)}(t)+c(t) \mu(t) q_{n+1}^{(m)}(t)+\mu(t) \sum_{j=n}^{c(t)}(c(t)-j) p_{j}(t) \prod_{k=1}^{m-2}(j-n+k)$,

where $\prod_{k=1}^{0} f(k)=1$. Define the generating functions

$$
\Psi_{z}^{(m)}(t)=\sum_{n=0}^{\infty} q_{n}^{(m)}(t) z^{n}
$$

Solving for the generating functions as in the previous section we have

$\Psi_{z}^{(1)}(t)=\left(1-\Phi_{z}(t-1, t)\right)^{-1} \int_{t-1}^{t} \Phi_{z}(\eta, t)\left(\lambda(\eta) q_{-1}^{(1)}(\eta)-c(\eta) \mu(\eta) z^{-1} q_{0}^{(1)}(\eta)+\mu(\eta) \sum_{n=0}^{c(\eta)}(c(\eta)-n) z^{n} p_{n}(\eta)\right) \mathrm{d} \eta$ 
and for $m>1$,

$$
\begin{aligned}
\Psi_{z}^{(m)}(t)= & \left(1-\Phi_{z}(t-1, t)\right)^{-1} \int_{t-1}^{t} \Phi_{z}(\eta, t)\left(\lambda(\eta) q_{-1}^{(m)}(\eta)-c(\eta) \mu(\eta) z^{-1} q_{0}^{(m)}(\eta)\right. \\
& \left.+\mu(\eta) \sum_{n=0}^{c(\eta)}(c(\eta)-n) p_{n}(\eta) \sum_{j=0}^{n} z^{j} \prod_{k=1}^{m-2}(n-j+k)\right) \mathrm{d} \eta .
\end{aligned}
$$

The rising factorial moment $E\left[(Q(t)+1)^{\bar{m}}\right]=\Psi_{z=1}^{(m)}(t)=q_{0}^{(m+1)}(t)$, yielding the following result:

Theorem 3. For $\int_{t-1}^{t} \lambda(s) \mathrm{d} s<\int_{t-1}^{t} c(s) \mu(s) \mathrm{d} s$, the periodic asymptotic mth rising factorial moment, $E[(Q(t)+$ $\left.1)^{\bar{m}}\right]$, for a time-dependent multi-server queue length process $\{Q(t), t \geqslant 0\}$ with initial distribution given by $P\{Q(0)=$ $n\}=p_{n}(0)$, periodic arrival rate $\lambda(t)$, service rate $\mu(t)$ and number of servers $c(t)$ with period 1 is given by the formula:

$$
\begin{aligned}
E\left[(Q(t)+1)^{\bar{m}}\right]= & (M(t-1, t)-\Lambda(t-1, t))^{-1} \int_{t-1}^{t}(\Lambda(\eta, t)-M(\eta, t))\left(\lambda(\eta) q_{-1}^{(m)}(\eta)\right. \\
& \left.-c(\eta) \mu(\eta) q_{0}^{(m)}(\eta)+\mu(\eta) \sum_{n=0}^{c(\eta)}(c(\eta)-n) p_{n}(\eta) \frac{(n+m-1) !}{n !(m-1)}\right) \\
& +c(\eta) \mu(\eta) q_{0}^{(m)}(\eta)+\mu(\eta) \sum_{n=0}^{c(\eta)}(c(\eta)-n) p_{n}(\eta) \frac{n(n+m-1) !}{m(m-1)(n !)} \mathrm{d} \eta
\end{aligned}
$$

for $m>1$, and

$$
\begin{aligned}
E[Q(t)+1]= & (M(t-1, t)-\Lambda(t-1, t))^{-1} \int_{t-1}^{t}[(\Lambda(\eta, t) \\
& -M(\eta, t))\left(\lambda(\eta)-c(\eta) \mu(\eta)+\mu(\eta) \sum_{n=0}^{c(\eta)}(c(\eta)-n) p_{n}(\eta)\right) \\
& \left.+c(\eta) \mu(\eta)+\mu(\eta) \sum_{n=0}^{c(\eta)}(c(\eta)-n) n p_{n}(\eta)\right] \mathrm{d} \eta,
\end{aligned}
$$

for $m=1$.

We may follow the same sequence of steps as in the derivation of (9) to rewrite these equations in terms of $p_{\hat{c}-1}(t)$, or we may follow the steps outlined in the previous section to first obtain the $p_{j}(t)$ and then solve for $E[Q(t)]$. The expectation and variance of the number in the queue are given by

$$
E[Q(t)]=\Psi_{z=1}^{(1)}(t)-1
$$

and

$$
\begin{aligned}
\operatorname{Var}[Q(t)] & =2 E\left[(Q(t)+1)^{\overline{2}}\right]-E[Q(t)+1]^{2}-E[Q(t)+1] \\
& =\Psi_{z=1}^{(2)}(t)-\left(\Psi_{z=1}^{(1)}(t)\right)^{2}-\Psi_{z=1}^{(1)}(t),
\end{aligned}
$$


respectively. In the corollary that follows we provide two simple formulae for $E[Q(t)]$ in terms of the probabilities $p_{j}(t), j=0, \ldots, c(t)-1$.

Corollary 1. For $\int_{t-1}^{t} \lambda(s) \mathrm{d} s<\int_{t-1}^{t} c(s) \mu(s) \mathrm{d} s$, the periodic asymptotic expected number for a time-dependent multi-server queue length process $\{Q(t), t \geqslant 0\}$ with initial distribution given by $P\{Q(0)=n\}=p_{n}(0)$, periodic arrival rate $\lambda(t)$, service rate $\mu(t)$ and number of servers $c(t)$ with period 1 is

$$
\begin{aligned}
E[Q(t)]= & \frac{\int_{t-1}^{t} \mu(\eta) \sum_{n=0}^{c(\eta)}(c(\eta)-j) j p_{j}(\eta) \mathrm{d} \eta}{M(t-1, t)-\Lambda(t-1, t)}+\frac{\Lambda(t-1, t)}{M(t-1, t)-\Lambda(t-1, t)} \\
& +\frac{\int_{t-1}^{t} \mu(\eta) \sum_{n=0}^{c(\eta)}(c(\eta)-j) p_{j}(\eta)(M(t-1, \eta)-\Lambda(t-1, \eta)+\Lambda(\eta, t)-M(\eta, t)) \mathrm{d} \eta}{2(M(t-1, t)-\Lambda(t-1, t))}, \\
E[Q(t)]= & \frac{\int_{t-1}^{t} \mu(\eta) \sum_{n=0}^{c(\eta)}(c(\eta)-j) j p_{j}(\eta) \mathrm{d} \eta}{M(t-1, t)-\Lambda(t-m a t h r m 1, t)}+\frac{\Lambda(t-1, t)}{M(t-1, t)-\Lambda(t-1, t)} \\
& +\frac{\int_{t-1}^{t} \mu(\eta) \sum_{n=0}^{c(\eta)}(c(\eta)-j) p_{j}(\eta)(\Lambda(\eta, t)-M(\eta, t)) \mathrm{d} \eta}{M(t-1, t)-\Lambda(t-1, t)}+\frac{M(t-1, t)-\Lambda(t-1, t)}{2} .
\end{aligned}
$$

When $\lambda, \mu$ and $c$ are constant, the term on the second line evaluates to zero.

\section{Examples}

We consider examples with time-varying periodic arrival rates for a single-server queue, a two-server queue and a queue which alternates between one and three servers. For the one and two-server queues, we show the simpler form taken by the integral equations needed to solve for $p_{\hat{c}-1}(t)$.

\subsection{A single server queиe}

For a single server queue, Eq. (9) becomes $p_{0}(t)=\int_{t-1}^{t} \mu(\eta) p_{0}(\eta)\left(H_{0}(\eta, t)-H_{1}(\eta, t)\right) \mathrm{d} \eta$, and Eq. (10) $M(t-1$, $t)-\Lambda(t-1, t)=\int_{t-1}^{t} \mu(\eta) p_{0}(\eta) \mathrm{d} \eta$. We consider a single server queue with constant service and time-varying arrival rate: $\mu(t)=\mu=3 \alpha, \lambda(t)=2 \alpha(1+\sin (2 \pi t))$, and $c(t)=c=1$, where $\alpha$ is a parameter. For this particular single server queue, $M(t-1, t)=3 \alpha$, and $\Lambda(t-1, t)=2 \alpha$, so $\int_{t-1}^{t} p_{0}(\eta) \mathrm{d} \eta=\frac{1}{3}$. See Figs. 1 and 2 .

\subsection{A two-server queue with sinusoidal arrivals}

We consider a two-server queue with time-varying arrival and service rates. For the two-server queue, $\mathbf{G}(s, t)$ simplifies to $\mathrm{e}^{-\Lambda(s, t)} /\left(1-\mathrm{e}^{-\Lambda(t-1, t)}\right)$. For our example, we consider a two-server queue with constant service and sinusoidal arrival rate. We consider a queue with arrival and service rates: $\lambda(t)=4(1+\sin (2 \pi t)), \mu(t)=\mu=3$, and $c(t)=c=2$. For this example, $\Lambda(s, t)=4(t-s+(1 / 2 \pi)(\cos (2 \pi s)-\cos (2 \pi t))), M(s, t)=6(t-s)$, and $M(t-1, t)-\Lambda(t-1, t)=2$, and $p_{1}(t)$ satisfies the equations (see Figs. 3 and 4 )

$$
\begin{aligned}
p_{1}(t)= & 3 \int_{t-1}^{t} p_{1}(\eta)\left[\left(H_{0}(\eta, t)-2 H_{1}(\eta, t)\right)+\frac{4}{1-\mathrm{e}^{-4}} \int_{t-1}^{\eta} \mathrm{e}^{-\Lambda(\eta-1, v)}(1+\sin (2 \pi v))\right. \\
& \left.\times H_{0}(v, t) \mathrm{d} v+\frac{4}{1-\mathrm{e}^{-4}} \int_{\eta}^{t} \mathrm{e}^{-\Lambda(\eta, v)}(1+\sin (2 \pi v)) H_{0}(v, t) \mathrm{d} v\right] \mathrm{d} \eta
\end{aligned}
$$




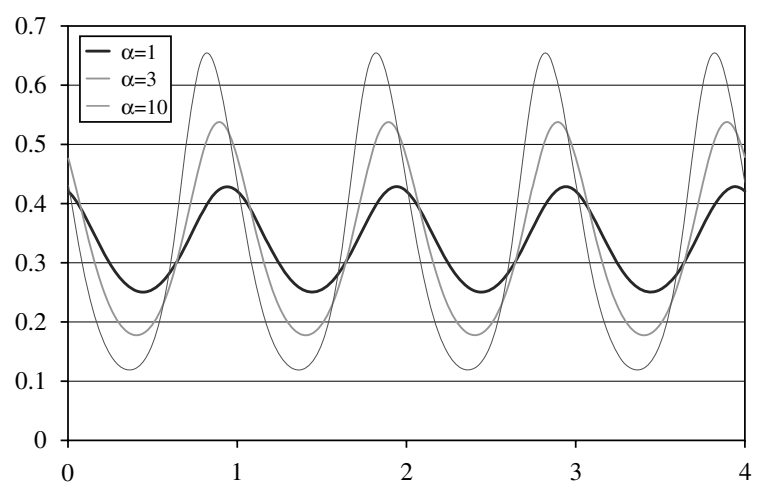

Fig. 1. Graphed are the idle probabilities for $\alpha=1,3,10$ for an $M_{t} / M / 1$ queue with arrival rate $\lambda(t)=2 \alpha(1+\sin (2 \pi t))$, service rate $\mu=3 \alpha$.

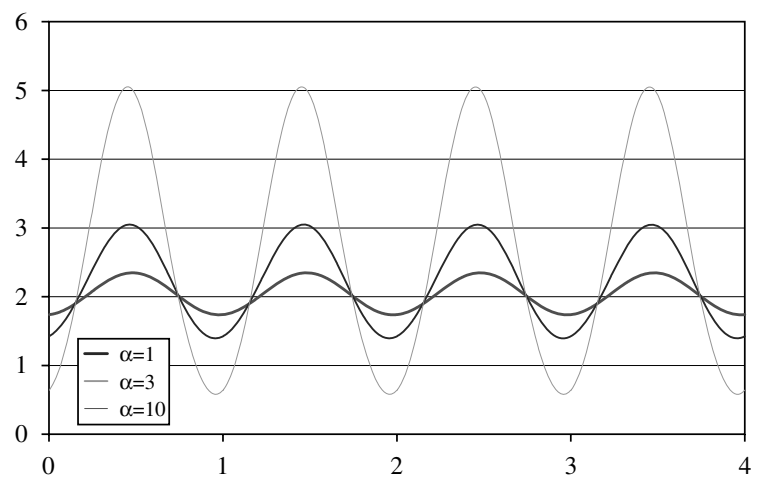

Fig. 2. Graphed is the expected number in the queue for $\alpha=1,3,10$ for an $M_{t} / M / 1$ queue with arrival rate $\lambda(t)=2 \alpha(1+\sin (2 \pi t))$, service rate $\mu=3 \alpha$. For a single server queue with constant rates $\lambda=2 \mu / 3$, the expected number in the queue is 2 .

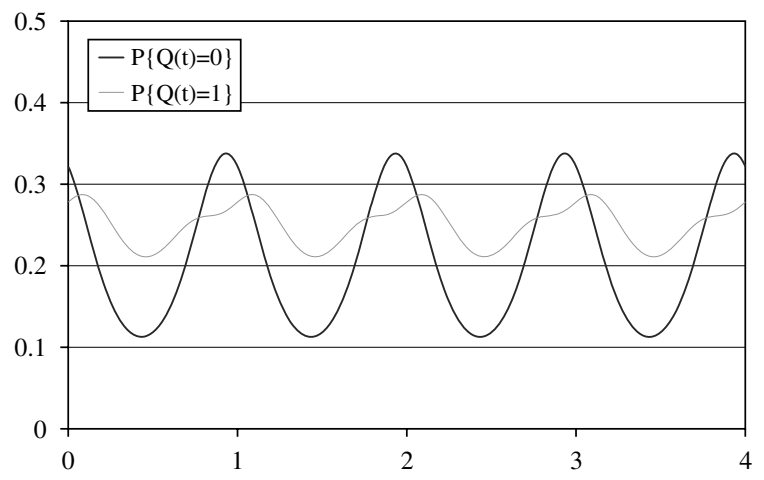

Fig. 3. Probability of zero or one in queue for a two-server queue with $\lambda(t)=4(1+\sin (2 \pi t)), \mu=3$. For a constant rate queue with the same mean rate values, $p_{0}=\frac{1}{5}$ and $p_{1}=\frac{4}{15}$. 


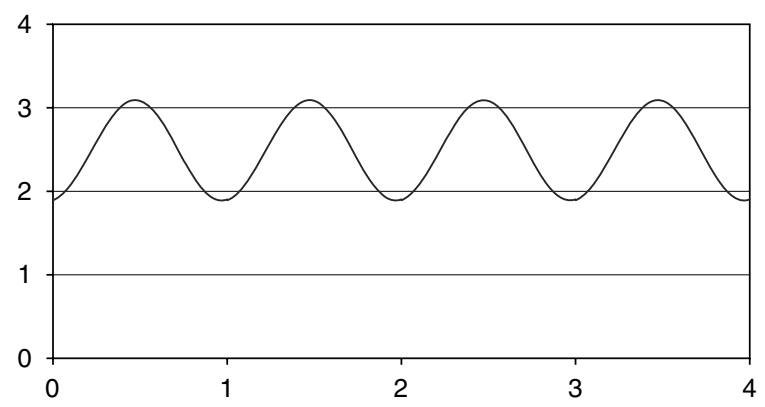

Fig. 4. Expected number in queue for a two-server queue with $\lambda(t)=4(1+\sin (2 \pi t)), \mu=3$. For a constant rate queue with the same mean rate values, $E[Q(t)]=\frac{12}{5}$.

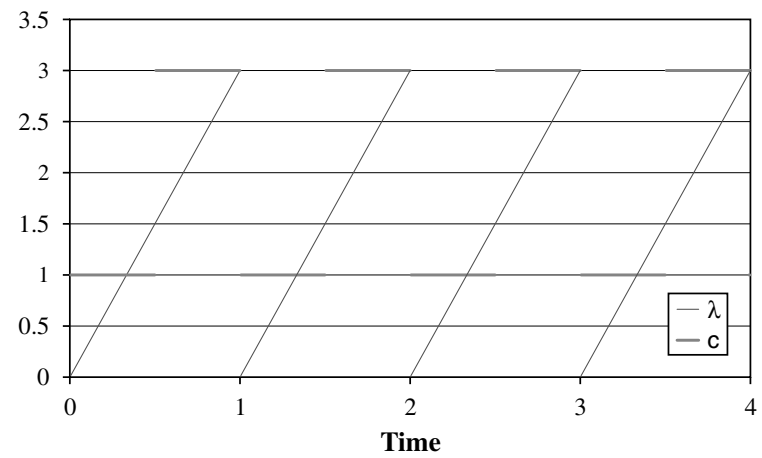

Fig. 5. Graphed are the arrival rate, $\lambda(t)=3(t-\lfloor t\rfloor)$, and number of servers, $c(t)$.

and

$$
\frac{\left(1-\mathrm{e}^{-4}\right)}{9}=\int_{t-1}^{t} p_{1}(\eta)\left[\frac{1-\mathrm{e}^{-4}}{6}+\int_{t-1}^{\eta} \mathrm{e}^{-\Lambda(\eta-1, v)} \mathrm{d} v+\int_{\eta}^{t} \mathrm{e}^{-\Lambda(\eta, v)} \mathrm{d} v\right] \mathrm{d} \eta
$$

\subsection{A three-server queue with sawtooth arrivals and time-varying number of servers}

We consider a queue with a single server during the first half of each period and three servers during the second half. Arrivals occur according to the sawtooth function $\lambda(t)=3(t-\lfloor t\rfloor)$. The service rate is constant at 1 , see Figs. 5-7.

\section{Conclusion}

Although the formulas given can be quite complicated, the method requires only one period of data so that periodic asymptotic results may be found without having to truncate an infinite system of differential equations, and without having to compute transient solutions over a long time horizon. Properties of modified Bessel functions may be exploited to provide estimates for the kernel functions in the integral equations derived herein. The formulae for the rising factorial moments of the periodic queue length process have a particularly simple structure once the $p_{j}(t)$ are known for $j$ less than the number of servers. 


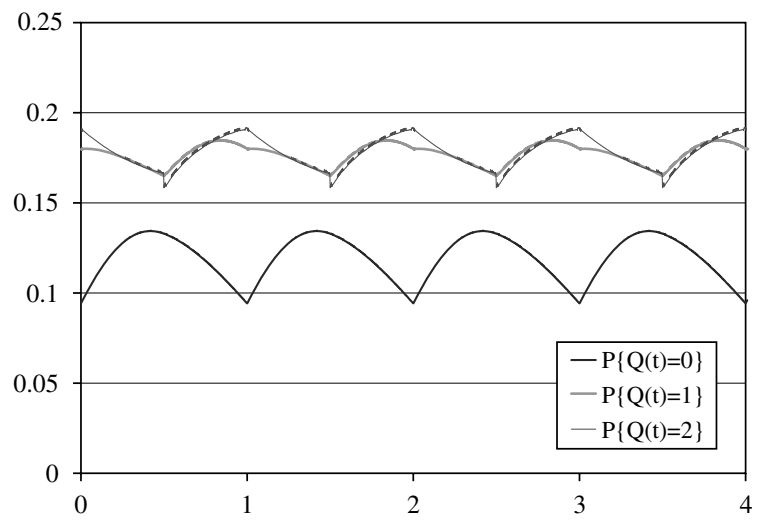

Fig. 6. Graphed are the probabilities of zero, one or two in the queue for a queue that alternates between one and three servers.

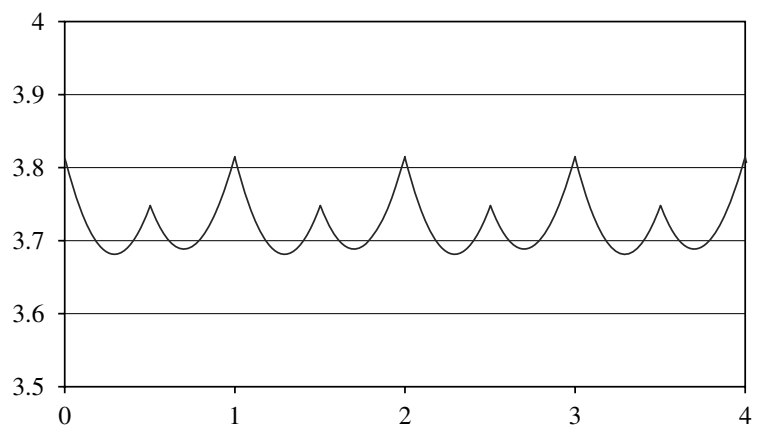

Fig. 7. The expected number in the queue for a queue that alternates between one and three servers.

The method may be extended to a broader class of continuous time Markov chains without change provided that after $k$ initial states, the chain has the same arrival rate, $\lambda_{n}(t)=\lambda_{k}(t)$, and departure rate $\mu_{n}(t)=\mu_{k}(t), n \geqslant k$. In particular, this approach could be used to model queues with heterogeneous servers.

\section{Acknowledgements}

This paper is part of a cooperative effort between Cleveland State University and the City of Cleveland Division of Police. In particular, I want to thank Deputy Chief Prioleau Green of the Police Division, and Felipe Martins, John Holcomb and Teresa LaGrange at Cleveland State University.

\section{Appendix A}

In this appendix, we derive a formula for estimating $H_{n}(\eta, t)$ and establish error bounds on the estimates.

Theorem 4. The quantity $H_{n}(\eta, t)$ may be estimated with a finite sum of random walk probabilities:

$$
H_{n}(\eta, t)=\sum_{j=0}^{N-1} \tilde{I}_{n, j}(\eta, t)+R_{1}(N, k, \eta, t),
$$


where

$$
0<R_{1}(N, k, \eta, t) \leqslant \frac{\tilde{I}_{k, N}(\eta, t)}{1-\mathrm{e}^{-\Lambda(t-1, t)-M(t-1, t)+2 \sqrt{M(t-1, t) \Lambda(t-1, t)}} .}
$$

Proof. Define: $R_{1}(N, k, \eta, t)=\sum_{j=N}^{\infty} \tilde{I}_{k, j}(\eta, t)$. Since each of the random walk probabilities is positive, it is clear that $R_{1}>0$. From the Chapman-Kolmogorov equations for a time-dependent random walk,

$$
R_{1}(N, k, \eta, t)=\sum_{j=N}^{\infty} \sum_{n=-\infty}^{\infty} \tilde{I}_{k-n}^{(j)}(t-1, t) \tilde{I}_{n}(\eta, t) .
$$

From [2, Eqs. (8.411), (8.406(3))], we have

$$
I_{n}(z)=\frac{i^{-n}}{2 \pi} \int_{-\pi}^{\pi} \mathrm{e}^{-n i \theta-z \sin \theta} \mathrm{d} \theta,
$$

so

$$
\tilde{I}_{n}(s, t)=\left(\frac{\Lambda(s, t)}{M(s, t)}\right)^{n / 2} \mathrm{e}^{-\Lambda(s, t)-M(s, t)} \frac{i^{-n}}{2 \pi} \int_{-\pi}^{\pi} \mathrm{e}^{-n i \theta-2 \sqrt{M(s, t) \Lambda(s, t)} \sin \theta} \mathrm{d} \theta .
$$

Using (14), and then changing the order of integration and summation in (13),

$$
\begin{aligned}
R_{1}(N, k, \eta, t)= & \frac{i^{-k}}{4 \pi^{2}} \sum_{n=-\infty}^{\infty}\left(\frac{\Lambda(t-1, t)}{M(t-1, t)}\right)^{(k-n) / 2}\left(\frac{\Lambda(\eta, t)}{M(\eta, t)}\right)^{n / 2} \mathrm{e}^{-\Lambda(\eta, t)-M(\eta, t)} \\
& \times \int_{-\pi}^{\pi} \int_{-\pi}^{\pi}\left[\mathrm{e}^{-i(k-n) \theta-n i \psi-2 \sqrt{M(\eta, t) \Lambda(\eta, t)} \sin \psi}\right. \\
& \left.\times \sum_{j=N}^{\infty} \mathrm{e}^{-j \Lambda(t-1, t)-j M(t-1, t)-2 j \sqrt{M(t-1, t) \Lambda(t-1, t)} \sin \theta}\right] \mathrm{d} \theta \mathrm{d} \psi .
\end{aligned}
$$

Since, $\Lambda+M+2 \sqrt{\Lambda M} \sin \theta \geqslant(\sqrt{\Lambda}-\sqrt{M})^{2},\left|\mathrm{e}^{-\Lambda-M-2 \sqrt{\Lambda M} \sin \theta}\right|<1$, we may sum the geometric series and obtain,

$$
\begin{aligned}
R_{1}(N, k, \eta, t)= & \frac{i^{-k}}{4 \pi^{2}} \sum_{n=-\infty}^{\infty}\left(\frac{\Lambda(t-1, t)}{M(t-1, t)}\right)^{(k-n) / 2}\left(\frac{\Lambda(\eta, t)}{M(\eta, t)}\right)^{n / 2} \mathrm{e}^{-\Lambda(\eta, t)-M(\eta, t)} \\
& \times \int_{-\pi}^{\pi} \int_{-\pi}^{\pi}\left[\mathrm{e}^{-i(k-n) \theta-n i \psi-2 \sqrt{M(\eta, t) \Lambda(\eta, t)} \sin \psi}\right. \\
& \times \frac{\mathrm{e}^{-N \Lambda(t-1, t)-N M(t-1, t)-2 N \sqrt{M(t-1, t) \Lambda(t-1, t)} \sin \theta}}{\left.1-\mathrm{e}^{-\Lambda(t-1, t)-M(t-1, t)-2 \sqrt{M(t-1, t) \Lambda(t-1, t)} \sin \theta}\right] \mathrm{d} \theta \mathrm{d} \psi .}
\end{aligned}
$$

Now

$$
\mathrm{e}^{-\Lambda(t-1, t)-M(t-1, t)-2 \sqrt{M(t-1, t) \Lambda(t-1, t)} \sin \theta} \leqslant \mathrm{e}^{-\Lambda(t-1, t)-M(t-1, t)+2 \sqrt{M(t-1, t) \Lambda(t-1, t)}},
$$


for all $\theta$, so using the integral formula for $\sum_{n=-\infty}^{\infty} \tilde{I}_{N, k-n}(t-1, t) \tilde{I}_{n}(\eta, t)$, we can see that,

$$
R_{1}(N, k, \eta, t) \leqslant \frac{\tilde{I}_{k, N}(\eta, t)}{1-\mathrm{e}^{-\Lambda(t-1, t)-M(t-1, t)+2 \sqrt{M(t-1, t) \Lambda(t-1, t)}}},
$$

as required. Because $\lim _{N \rightarrow \infty} \tilde{I}_{N, k}(\eta, t)=0$, we may approximate $H_{n}(\eta, t)$ with a finite sum of the random walk probabilities $\tilde{I}_{k, j}(\eta, t)$.

\section{References}

[1] L. Breuer, The periodic BMAP/PH/c queue, Queueing Syst. 38 (2001) 67-76.

[2] I.S. Gradshteyn, I.M. Ryzhik, in: A. Jeffries (Ed.), Table of Integrals, Series, and Products, fifth ed., Academic Press, 1994.

[3] A.M. Hasofer, On the integrability, continuity and differentiability of a family of functions introduced by L. Takàcs, Ann. Math. Stat. 34 (1963) 1045-1049.

[4] A.M. Hasofer, On the single-server queue with non-homogeneous Poisson input and general service time, J. Appl. Probab. 1 (1964) 369-384.

[5] A. Ingolfsson, Modeling the $M(t) / M / s(t)$ queue with an exhaustive discipline, Working paper, available online at $\langle$ http://www.bus. ualberta.ca/aingolfsson/working_papers.htm. $\rangle, 2005$.

[6] D.E. Knuth, Two notes on notation, Am. Math. Mon. 99 (1992) 403-422.

[7] A.N. Kolmogorv, Sur le probleme d'attente, Mat. Sb. 38 (1931) 101 (French).

[8] A.N. Kolmogorv, The waiting problem, in: A.N. Shiryayev (Ed.), Selected Works of A.N. Kolmogorov, vol. II: Probability Theory and Mathematical Statistics, Kluwer, Boston, 1991, pp. 109-114 (translated by G. Lindquist).

[9] E.L. Leese, D.W. Boyd, Numerical methods of determining the transient behavior of queues with variable arrival rates, J. Can. Oper. Res. Soc. 4 (1966) 1-13.

[10] B.H. Margolius, Transient solution to the time-dependent multi-server Poisson queue, J. Appl. Probab. 42 (2005) 766-777.

[11] W.A. Massey, The analysis of queues with time-varying rates for telecommunication models, Telecommun. Syst. 21 (2002) 173-204.

[12] D.L. Minh, The discrete-time single-server queue with time-inhomogeneous compound Poisson input and general service time distribution, J. Appl. Probab. 15 (1978) 590-601.

[13] N.J.A. Sloane, The On-Line Encyclopedia of Integer Sequences, published electronically at 〈http://www.research.att.com/ njas/ sequences/), 〈http://www.research.att.com/projects/OEIS?Anum = A000254.〉, 2005.

[14] H.S. Wilf, Generating Functionology, second ed., Academic Press, Boston. Also available online at /http://www.math.upenn.edu/ $\sim$ wilf/DownldGF.html $>, 1994$.

[15] G. Yin, H. Zhang, Two-time-scale Markov chains and applications to quasi-birth-death queues, SIAM J. Appl. Math. 65 (2) (2005) $567-586$.

[16] J. Zhang, E.J. Coyle, The transient solution of time-dependent M/M/1 queues, IEEE Trans. Inf. Theory 37 (6) (1991) 1690-1696.

Post-print standardized by MSL Academic Endeavors, the imprint of the Michael Schwartz Library at Cleveland State University, 2017 